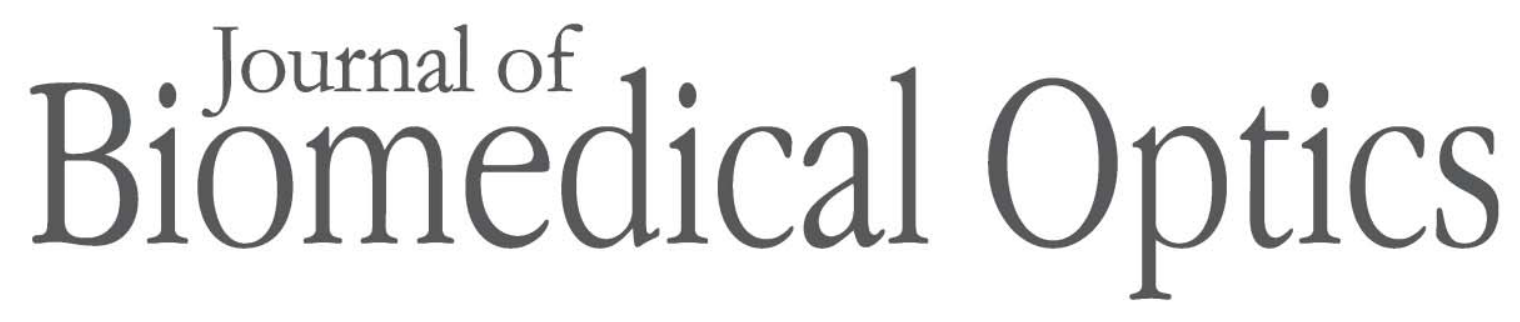

SPIEDigitalLibrary.org/jbo

\title{
Red-shifted fluorescence of sound dental hard tissue
}

Liang Zhang

Leonard Y. Nelson

Eric J. Seibel 


\title{
Red-shifted fluorescence of sound dental hard tissue
}

\author{
Liang Zhang, ${ }^{a}$ Leonard Y. Nelson, ${ }^{b}$ and Eric J. Seibel ${ }^{b}$ \\ a University of Washington, Department of Bioengineering, Seattle, Washington 98195 \\ buniversity of Washington, Department of Mechanical Engineering, Seattle, Washington 98195
}

\begin{abstract}
Autofluorescence spectra were recorded in vitro from dentin, enamel, and whole teeth. The spectra exhibited a broad peak shifted by about 50 to $75 \mathrm{~nm}$ from the excitation wavelength and the shape of the spectra remained similar regardless of the excitation wavelength. The maximum of the autofluorescence spectra also exhibited a red-shift that depended upon the laser excitation wavelength. The amplitude of the red-shifted fluorescence spectra produced by 444 and $532 \mathrm{~nm}$ excitation lasers were compared to that produced by a $405 \mathrm{~nm}$ excitation laser. It was determined that the autofluorescence amplitude was not proportional to the inverse fourth power of the excitation laser wavelength. Therefore, the red-shifted fluorescence is not compatible with the previously proposed mechanism of Raman scattering. Instead, the mechanism giving rise to the laser-induced dental autofluorescence is explained by the red-edge-excitation effect. $\odot 2011$ Society of Photo-Optical Instrumentation Engineers (SPIE). [DOI: 10.1117/1.3606572]
\end{abstract}

Keywords: autofluorescence; red-edge-excitation shift; Raman scattering; collagen; 3-hydroxypyridinium; pyridinoline; enamel; dentin. Paper 11070SSRR received Feb. 14, 2011; revised manuscript received Jun. 13, 2011; accepted for publication Jun. 13, 2011; published online Jul. 21, 2011.

\section{Introduction}

Autofluorescence is based on the presence of endogenous fluorophores residing in the enamel and dentin. ${ }^{1,2}$ It is well known that dental enamel and dentin exhibit laser-induced fluorescence at wavelengths longer than the excitation wavelength. Among the earliest reports of dental autofluorescence is the detection of an orange and red fluorescence of the "tooth film" in patients. ${ }^{3}$ Later, tooth autofluorescence was observed under ultraviolet excitation. ${ }^{4,5}$ Since then, there have been many studies on dental autofluorescence and it has now become a diagnostic tool in caries detection (QLF, Inspektor Research Systems, Netherlands).

The fluorescence spectra of sound teeth have been reported to be dependent on excitation wavelength. ${ }^{6}$ A red-shift of the excitation wavelength led to a corresponding red-shift in the wavelength of maximum autofluorescence. This phenomenon will henceforth be defined as "red-shifted autofluorescence." Therefore, the governing mechanism of dental autofluorescence does not obey classical fluorescence, where the peak fluorescence wavelength is largely independent of excitation wavelength. ${ }^{7}$ However, the mechanism governing the observed phenomenon is still unclear. Therefore, understanding the fluorescence emission mechanism of tooth enamel and dentin will be fundamental toward the development of further autofluorescence-based dental diagnostic technologies.

In the present study, quantitative fluorescence measurements of dental specimens were obtained. Specimens were excited at four laser wavelengths $(405,444,532$, and $635 \mathrm{~nm})$, and absolute intensity ratios were measured. These results were analyzed to determine whether Raman scattering ${ }^{6}$ was responsible for the red-shifted spectra.

Address all correspondence to: Eric Seibel, University of Washington, Departmen of Mechanical Engineering, Human Photonics Laboratory, 204 Fluke Hall, Box 352142, Seattle, Washington 98195-2142; Tel: 206-616-1486; Fax: 206-6858047; E-mail: eseibel@u.washington.edu.

\section{Materials and Methods}

\subsection{Specimen Preparation}

The Institutional Review Board approved a study that used sound human extracted molars and premolars $(n=14), 10$ intact specimens, 2 sectioned coronal dentin specimens, and 2 sectioned enamel specimens, all with surfaces gently cleaned by a toothbrush and were stored in a $0.1 \%$ thymol solution at room temperature. The dentin specimens were obtained by laterally sectioning through the center of two sound teeth. The enamel specimen was cut off the teeth and ground from the dentin-enamel junction until only enamel remained. Absence of lesions was confirmed by using visual inspection as well as the QLF dental diagnostic tool (Inspektor Research Systems, Netherlands). ${ }^{8}$ The surfaces of the specimens were cleaned via a toothbrush, but left unpolished and curved to represent clinical situations. ${ }^{8}$

\subsection{Instrumentation}

Emission spectra were measured using a commercial spectrometer with a grating groove density of 600 lines $/ \mathrm{mm}(650 \mathrm{~nm}$ spectral range) and a $200 \mu \mathrm{m}$ width entrance aperture (USB $2000+$, Ocean Optics Inc., Dunedin, Florida). It was fitted with a 2048 element linear silicon CCD detector array (ILX511, Sony Corp., Tokyo, Japan) with a corrected linearity of greater than $99.8 \%$. The computed resolution of the spectrometer was $4.8 \mathrm{~nm}$.

The experimental setup is shown schematically in Fig. 1. It consists of a fiber coupled diode laser module (FTEC2, Blue Sky Research, Milpitas, California) containing a 405nm laser diode (NDV4313, Nichia Corp., Tokushima, Japan), 444-nm fiber coupled diode laser module (FTEC2, Blue Sky Research, Milpitas, California) containing a blue laser diode (NDHB510APA, Nichia Corp., Tokushima, Japan), a 532-nm fiber coupled diode laser module (FTEC2, Blue

1083-3668/2011/16(7)/071411/5/\$25.00 (C) 2011 SPIE 


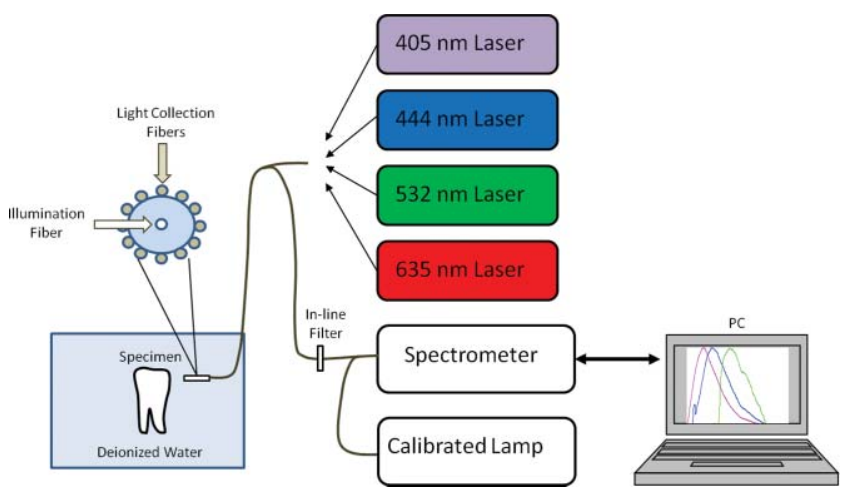

Fig. 1 Schematic diagram of the experimental setup. The extracted teeth were held in a chamber filled with water. A custom flexible fiber illuminated the teeth enamel with either a 405, 444, 532, or $635 \mathrm{~nm}$ laser. The collected fluorescence passed through an in-line optical filter to remove laser light before entering the spectrometer. The spectra were saved on the PC and analyzed offline.

Sky Research, Milpitas, California), and a 635-nm fiber coupled diode laser module (FTEC2, Blue Sky Research, Milpitas, California). All lasers were measured with the spectrometer to confirm the laser wavelength. A 1.2-mm fiber bundle was assembled in our laboratory to guide the light from the lasers to the test specimens and deliver the autofluorescence to the spectrometer. ${ }^{9}$ A small diameter $(0.8 \mathrm{~mm})$ lens, not shown in Fig. 1, is positioned $150 \mu \mathrm{m}$ from the end of the laser excitation delivery fiber. The focused laser spot size at the specimen is approximately $25 \mu \mathrm{m}$.

\subsection{Experimental Procedure}

Continuous wave output power was set to a constant at $1.34 \mathrm{~mW}$ for all lasers using an optical power meter (Model 1835-c, Newport Corp., Irvine, California). A $420 \mathrm{~nm}$ in-line longpass filter (E420LPv2, Chroma Technology Corp., Bellows Falls, Vermont), or a $450 \mathrm{~nm}$ in-line longpass filter (NT62-982, Edmund Optics Inc., Barrington, New Jersey), or a $532 \mathrm{~nm}$ inline rejection filter (NT63-346, Edmund Optics Inc., Barrington, New Jersey), or a $645 \mathrm{~nm}$ in-line longpass filter (HQ645LP, Chroma Technology Corp., Bellows Falls, Vermont), were used individually for every excitation wavelength to attenuate excitation laser light at the spectrometer entrance aperture.

Teeth specimens were placed in a custom made chamber filled with deionized water. The fiber tip was placed in the chamber and submerged in the water. Fluorescence scans were performed for all four excitation lasers using a detector integration time of $100 \mathrm{~ms}$. All emission spectra were recorded in a dark room to reduce stray ambient light. A universal serial bus port connected the spectrometer to a computer for spectral recordings.

The spectral sensitivity of the spectrometer was corrected prior to data acquisition by a NIST-traceable (National Institute of Standards and Technology) calibrated tungsten halogen lamp (LS-1-CAL, Ocean Optics Inc., Dunedin, Florida). Additionally, the dark spectrum was measured and stored, which was then subtracted out during spectral acquisition to reduce noise and further ensure removal of stray light. The spectral transmission of the fluorescence isolation filters were measured with the spectrometer and the calibrated tungsten halogen lamp.

\subsection{Data Processing}

All emission spectra were filtered with a 13-point (corresponding to a spectral width of $4.94 \mathrm{~nm}$ ) median filter to remove electronic noise, followed by a 13-point Gaussian smoothing filter. For each sample, all emission spectra were normalized to the peak intensity of the emission spectra obtained using the 405-nm excitation laser, so that the peak intensity of the main peak of the $405 \mathrm{~nm}$ emission spectra was set to 1 . By normalizing the emission spectra, we were able to compare intensity values between different excitation lasers for each tooth, as well as for different teeth specimens. Additionally, another autofluorescence spectra plot was created where each spectrum was normalized to 1.0 to observe spectral shape.

The measured transmission values of the fluorescence isolation filters were included in the calculations of the peak fluorescence intensity ratios. To further investigate the autofluorescence, the ratios were also determined for the two sectioned dentin specimens and two sectioned enamel specimens.

\section{Results}

All 10 intact specimens exhibited autofluorescence for 405, 444, and $532 \mathrm{~nm}$ excitation wavelengths [Fig. 2(a)]. At $635 \mathrm{~nm}$, there was no detectable autofluorescence. The emission spectra showed a broad peak centered about 50 to $75 \mathrm{~nm}$ from the excitation wavelength, and gradually tapered off toward the longer wavelengths (peak fluorescence at 480, 512, and $579 \mathrm{~nm}$ for 405, 444, and $532 \mathrm{~nm}$ excitation, respectively). The shape of the spectra remained very similar regardless of the excitation wavelength [Fig. 2(b)], except that the autofluorescence intensity diminished greatly as the excitation wavelength increased [Fig. 2(a)]. The standard deviation of the peak fluorescence signal for 512 and $579 \mathrm{~nm}$ (corresponding to 444 and $532 \mathrm{~nm}$ excitation, respectively) normalized by $480 \mathrm{~nm}$ (corresponding to $405 \mathrm{~nm}$ excitation) is shown. The small peak left of the main peak visible in the 512 and $579 \mathrm{~nm}$ spectra is from the excitation laser reaching the spectrometer that was not fully attenuated by the optical filters.

As shown in Table 1, the intensity of the fluorescence signal for an intact specimen decreased by a factor of 4.27 as the excitation wavelength increased from 405 to $444 \mathrm{~nm}$. When the excitation wavelength changed from 444 to $532 \mathrm{~nm}$, the ratio became 7.18. Finally, when comparing the fluorescence intensity between 405 and $532 \mathrm{~nm}$ excitation, the intensity ratio was 30.59. It should be noted that the most sensitive ratio to examine when determining the wavelength dependence of the autofluorescence intensity is the one between the largest excitation wavelength differences, e.g., 532 and $405 \mathrm{~nm}$ (Table 1, row 3).

The peak fluorescence intensity ratios for pure enamel and pure dentin are also shown in Table 1. Autofluorescence from enamel and from dentin behave similar to the autofluorescence from the 10 intact teeth specimens.

\section{Discussion}

Photoexcited emission from enamel and dentin is well known and generally assigned to organic matter embedded in the inorganic calcium apatite semicrystalline matrix. ${ }^{10}$ Buchalla has reported detailed spectra of emission from cross-sectioned enamel 

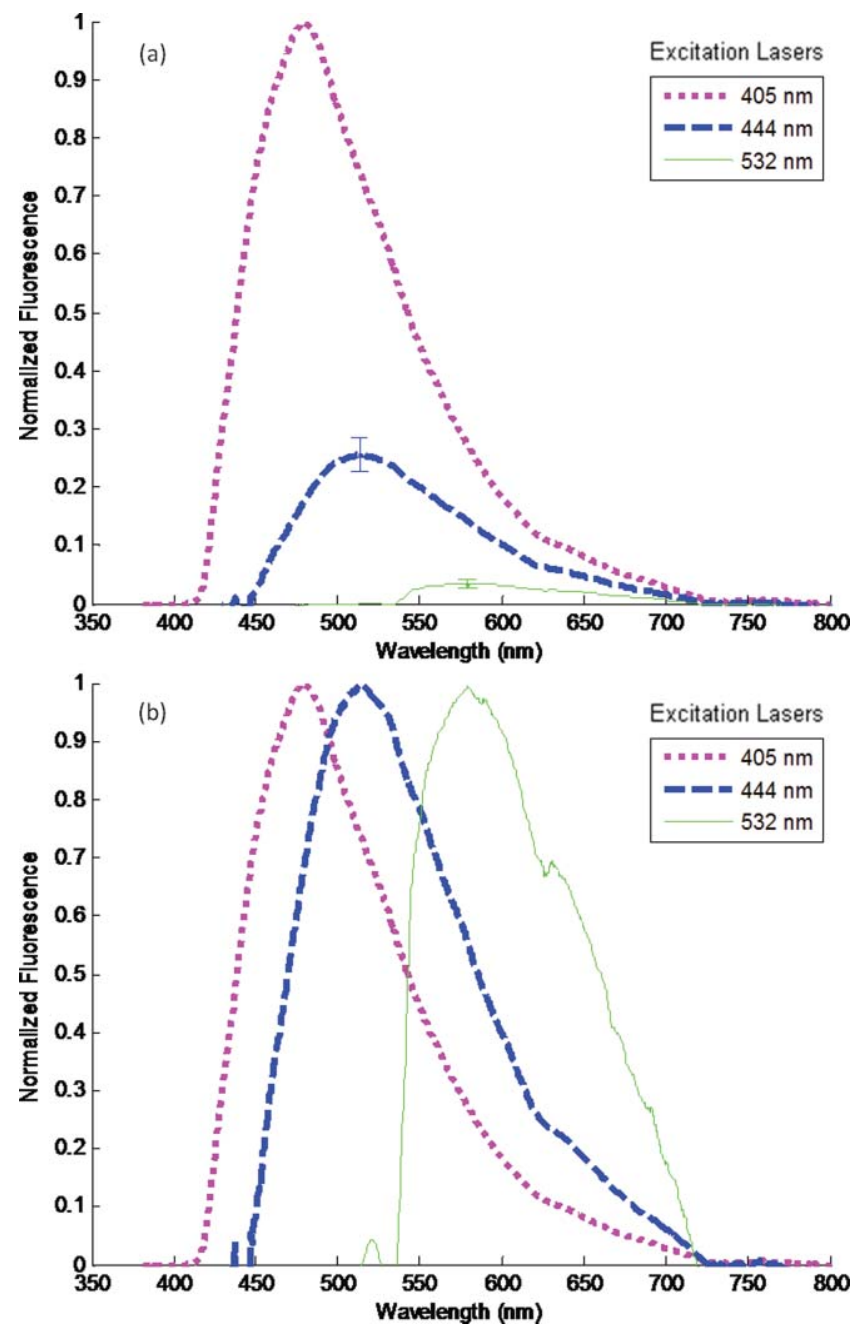

Fig. 2 Averaged emission spectra for intact sound teeth $(n=10)$ for 405, 444, and $532 \mathrm{~nm}$ excitation normalized by the peak intensity of the 405 emission spectra. (a) As excitation wavelength increased, fluorescent intensity decreased greatly. (b) Average emission spectra for each excitation wavelength normalized to their respective main peak.

samples subjected to a range (380 to $580 \mathrm{~nm}$ ) of excitation wavelengths. ${ }^{6} \mathrm{He}$ observed a red-shift of the peak emission wavelength as the excitation moved to a longer wavelength. In the present study, laser excitation of extracted teeth at three wavelengths $(405,444$, and $532 \mathrm{~nm})$ revealed the same

Table 1 Intensity ratios between the three excitation laser wavelengths.

\begin{tabular}{llrrr}
\hline Peak fluorescence & \multicolumn{1}{l}{$\begin{array}{l}\text { Whole } \\
\text { teeth }\end{array}$} & Enamel & Dentin & $\begin{array}{c}\text { Predicted } \\
\text { Raman } \\
\text { scattering }\end{array}$ \\
\hline F405/F444 & $4.27 \pm 0.44$ & 4.30 & 9.00 & 1.44 \\
F444/F532 & $7.18 \pm 1.69$ & 6.14 & 3.22 & 2.06 \\
F405/F532 & $30.59 \pm 5.75$ & 26.40 & 29.02 & 2.98 \\
\hline
\end{tabular}

red-shifted emission from sound enamel (Fig. 2) as found by Buchalla. As the excitation wavelength was moved from short wavelengths $(405 \mathrm{~nm})$ to longer wavelengths $(532 \mathrm{~nm})$, the peak of the observed emission shifted from 480 to $579 \mathrm{~nm}$, respectively.

Excitation from the ground state to an excited electronic state of a molecule generally follows the Franck-Condon principle and favors excited and ground states with strong overlap of their vibronic levels. In many cases (e.g., nonpolar fluorophore and homogeneous nonpolar solvent), the excited upper electronic state rapidly relaxes to its lowest energy level on a time scale much shorter (picoseconds) than the fluorescence lifetime (nanoseconds). This nonradiative relaxation produces an emission wavelength relatively independent of the excitation wavelength. This characteristic of fluorescent light emission is often referred to as "Kasha's Rule."

Therefore, to account for the unexpected wavelength shift of the enamel emission, Buchalla mentions as a possibility that the emission from sound specimens could be due to Raman scattering rather than fluorescence. However, if the emitted wavelengths are the result of Raman scattering, their relative intensity should be proportional to the fourth power of the inverse of the exciting wavelength. Hence, the ratio of the $500 \mathrm{~nm}$ emission to the $560 \mathrm{~nm}$ emission should be $(532 / 405)^{4}$, which equals 2.97 . However, this theoretical ratio is nearly 10 times smaller than the observed ratio found in this study (Table 1) and well outside the uncertainty range and, therefore, is not consistent with a Raman mechanism. In addition, a change from 405 to $635 \mathrm{~nm}$ excitation should lead to a factor of 6.04 decrease in the Raman scattering signal. However, no detectable signal was observed with $635 \mathrm{~nm}$ excitation. A factor of 6.04 decrease in signal should be easily detected by the spectrometer since a factor of 28.99 decrease was detected for $405 \mathrm{~nm}$ going to $532 \mathrm{~nm}$.

Furthermore, the strong Stokes shifted Raman emission feature from the phosphate component of the dental enamel was not observed in the Buchalla spectra nor in the present study. The phosphate narrow band emission should appear at $960 \mathrm{~cm}^{-1}$ Stokes shifted from the excitation wavelength: $421 \mathrm{~nm}$ for $405 \mathrm{~nm}$ excitation and $561 \mathrm{~nm}$ for $532 \mathrm{~nm}$ excitation. ${ }^{11}$ Finally, the observed emission intensities are qualitatively too strong for typical Raman scattering.

Environmental factors such as fluorophore and solvent polarity can alter the conventional pattern of fluorescent emission. The anisotropy observed in polarized fluorescence studies ${ }^{12}$ suggests a nonuniform environment surrounding the fluorophore in the enamel and dentin, which can produce a distribution of electronic energy levels for the fluorophore. Hence, a multitude of ground and excited states will exist throughout the matrix. The fluorophore in such a heterogeneous environment will behave as a collection of distinct species, and thus the spectroscopic properties of this ensemble will not be uniform. ${ }^{13,14}$

The absorption spectra of a heterogeneously distributed fluorophore will appear broad due to the spatially nonequivalent ground and excited states. Excitation on the red-edge of the absorption band will selectively excite a specific subpopulation of fluorophores, each with an electronic energy gap that matches the excitation wavelength and is a function of its local geometry. Additionally, if the timescale of obtaining a relaxed equilibrium between the fluorophore and environment is greater than the excited-state lifetime of the fluorophore, the fluorescence 

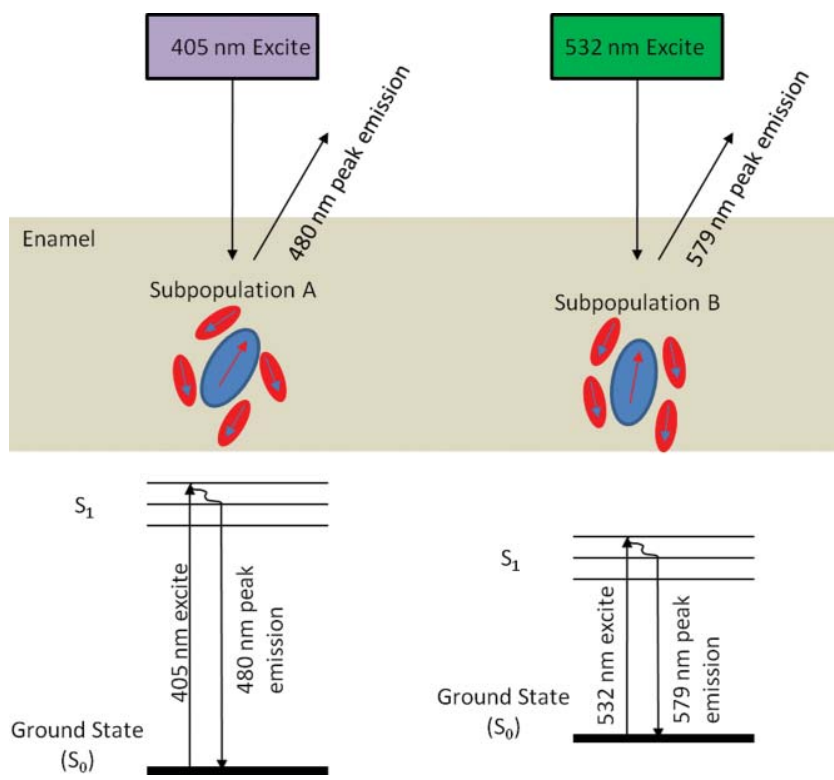

Fig. 3 Pictorial representation of red-edge-excitation shift. In the enamel or dentin, a heterogeneous distribution of fluorophore (large oval) and its calcium apatite environment (small ovals) exists. Arrows inside ovals indicate polarity. Different excitation wavelength lasers exciting on the red-edge of the absorption band of the fluorophore will selectively excite different subpopulations. In this example, $405 \mathrm{~nm}$ excites subpopulation $A$ and $532 \mathrm{~nm}$ excites subpopulation B, each with an electronic energy gap that matches the excitation wavelength. Excitation at progressively increasing wavelengths on the red-edge of the absorption curve will produce fluorescence at increasingly redshifted wavelengths if the time scale of obtaining a relaxed equilibrium between the fluorophore and environment is greater than the excitedstate lifetime of the fluorophore.

wavelength of these emitters will be unique to the selected sites due to its perturbed fluorophore/environment interaction (Fig. 3). This phenomenon is often denoted as the "red-edgeexcitation shift" (REES) or "red-edge effects." (Refs. 13 and 14). Site specific excitation of a polar fluorophore at progressively increasing wavelengths on the red-edge of the absorption curve will produce fluorescence at increasingly red-shifted wavelengths.

A likely fluorescent component of the enamel and dentin is an amino acid based on the 3-hydroxypyridinium ring and it is the major cross-linking element of collagens. ${ }^{15-17}$ There are two chemical forms, hydroxylysylpyridinoline (HP) and lysylpyridinoline (LP), that are based on the 3-hydroxypyridinium ring and both are naturally fluorescent. The hydroxypyridinium cross-link is present in most mature connective tissues, bone, and dentin, and has been recently found in enamel. ${ }^{18}$ Collagen derives its strength from packing into cross-linked fibrils, which reinforce bone, teeth, and tissue. As a result, the local environment of the HP and LP fluorophores is heterogeneous in dentin and enamel since the long chain collagen is embedded in a polycrystalline calcium apatite matrix and is partially surrounded by water.

The peak absorption of both HP and LP under neutral $p \mathrm{H}$ conditions is located at $325 \mathrm{~nm}$. Excitation at the peak absorption produces a broad ( $\sim 70 \mathrm{~nm}$ FWHM) fluorescence band centered near $400 \mathrm{~nm}$. Excitation at the peak absorption wavelength leads to a large $75 \mathrm{~nm}$ Stokes shift that is comparable to the red-shifts reported by Buchalla and observed in this study. Excitation of $\mathrm{HP}$ and LP at wavelengths beyond $350 \mathrm{~nm}$ meets the criteria for red-edge excitation.

This work does not conclusively demonstrate that HP and LP are the only fluorophores. Indeed, other fluorescent species have been proposed in the past, for example, tryptophan and tyrosine, and this work does not preclude their contribution to the observed fluorescence. ${ }^{1}$ In any case, a Raman scattering mechanism does not fit the red-shifted autofluorescence.

In the present study, the excitation laser was not localized to a particular depth in the whole tooth specimen. As the excitation laser wavelength increases, the depth of penetration also increases. ${ }^{19}$ Therefore, it is expected that excitation at 635 and $532 \mathrm{~nm}$ would not only interrogate enamel but likely contain a contribution from the enamel dentin junction as well as the dentin, whereas $405 \mathrm{~nm}$ would be more localized to the enamel. As such, $405 \mathrm{~nm}$ excitation should theoretically excite less fluorophor than the longer wavelengths because it is more highly attenuated. Therefore, penetration depth cannot explain the observed dramatic decrease in autofluorescence intensity with 635 and $532 \mathrm{~nm}$ excitation (Table 1).

It is known that scattering in dentin is relatively independent of wavelength. ${ }^{20,21}$ Therefore, if the mechanism governing the observed results is due to scattering, the spectra will not change with increasing excitation wavelengths in the dentin. The peak fluorescence intensity ratios for dentin are shown in Table 1. The autofluorescence from dentin and enamel behaved similar to autofluorescence from the 10 intact specimens. In all cases, the change in peak intensity decreased by a factor of 30 going from 405 to $532 \mathrm{~nm}$ excitation, whereas only a factor of 3 decrease would be expected from a Raman mechanism. Therefore, the observed relative intensities are not strongly influenced by scattering.

Laser-induced autofluorescence is a promising and exciting technology in the fight to detect early caries. Proper understanding of underlying mechanisms which govern the observed fluorescence will lead to greater insight and may permit successful detection of early carious lesions. This study demonstrated that dental autofluorescence intensity is heavily dependent on excitation wavelength, with an exponential decrease in emission intensity with increasing excitation wavelength, while the shape of the broad emission peak is relatively insensitive to the excitation wavelength. The governing mechanism which describes the results is provided by REES, where the heterogeneity of the fluorophore/environment population allows for different subpopulations to be excited in the red-edge of the absorption band, thus leading to an excitation wavelength dependent fluorescence spectrum.

\section{Acknowledgments}

The authors would like to thank Dr. Jim Wefel and the University of Iowa for the procurement and preparation of the intact teeth specimens used in this study. We appreciate Dr. Joel Berg at The Center for Pediatric Dentistry for his comments on the draft, and providing enamel specimens as well as Grant Beyer at The Center for Pediatric Dentistry for his assistance in this study. The study was partially funded from the University of Washington's Center for Commercialization, Washington Research Foundation, and the Life Sciences Discovery Fund. Liang Zhang 
would like to thank the University of Washington Department of Bioengineering in administrating the Cable Fellowship. The authors would also like to thank the reviewers for their constructive comments.

\section{References}

1. K. König, G. Flemming, and R. Hibst, "Laser-induced autofluorescence spectroscopy of dental caries," Cell Mol. Biol. 44, 1293-1300 (1998).

2. B. Angmar-Mansson and J. J. ten Bosch, "Optical methods for the detection and quantification of caries," Adv. Dent. Res. 1, 14-20 (1987).

3. S. Bommer, "Hautuntersuchungen im gefilterten quarzlicht," Klin. Wochenschr. 24, 1142-1144 (1927).

4. H. C. Benedict, "Note on the fluorescence of teeth in ultra-violet rays," Science 7, 70-79 (1928).

5. R. L. Hartles and A. G. Leaver, "The fluorescence of teeth under ultraviolet irradiation," Biochem. J. 54, 632-638 (1953).

6. W. Buchalla, "Comparative fluorescence spectroscopy shows differences in noncavitated enamel lesions," Caries Res. 39, 150-156 (2005).

7. J. R. Lakowicz, Principles of Fluorescence Spectroscopy, Springer, New York, NY (2006)

8. J. Wefel, Professor of pediatric dentistry, University of Iowa, Iowa City, Iowa, personal correspondence (2010).

9. C. M. Lee, C. J. Engelbrecht, T. D. Soper, F. Helmchen, and E. J. Seibel, "Scanning fiber endoscopy with highly flexible, $1 \mathrm{~mm}$ catheterscopes for wide-field, full-color imaging," J. Biophoton. 3(5-6), 385-407 (2010).

10. F. Sundström, K. Fredriksson, S. Montan, U. Hafström-Bjork-Man, and J. Ström, "Laser-induced fluorescence from sound and carious tooth substance: spectroscopic studies," Swed. Dent. J. 9, 71-80 (1985).
11. J. F. Freeman and M. J. Silva, "Separation of the Raman spectral signatures of bioapatite and collagen in compact mouse bone bleached with hydrogen peroxide," Appl. Spectrosc. 56, 770-775 (2002).

12. Y. Fukishima, T. Araki, and M. Yamada, "Topography of fluorescence and its possible composites in human teeth," Cell. Mol. Biol. 33, 725-736 (1987)

13. W. C. Galley and R. M. Purkey, "Role of heterogeneity of the salvation site in electronic spectra in solution," Proc. Nat. Acad. Sci. U.S.A. 67, 1116-1121 (1970).

14. A. P. Demchenko, "The red-edge effects: 30 years of exploration," $J$. Lumine. 17, 19-42 (2002).

15. D. R. Eyre, "Cross-linking in collagen and elastin," Ann. Rev. Biochem. 53, 717-748 (1984).

16. S. S. Thomas, S. Mohanty, J. L. Jayanthi, J. M. Varughese, A. Balan, and N. Subhash, "Clinical trial for detection of dental caries using laser-induced fluorescence ratio reference standard," J. Biomed. Opt. 15, $027001(2010)$

17. E. M. Rivera and M. Yamauchi, "Site comparisons of dentine collagen cross-links from extracted human teeth," Archs. Oral. Biol. 38, 541-546 (1993).

18. Y. Açil, A. E. Mobasseri, P. H. Warnke, H. Terheyden, J. Wiltfang, and I. Springer, "Detection of mature collagen in human dental enamel," Calcif. Tissue Int. 76, 121-126 (2005).

19. R. H. W Brodbelt, W. J. O’Brien, P. L. Fan, J. G. Frazer-Dib, and R. Yu, "Translucency of human dental enamel," J. Dent. Res. 60, 1749-1753 (1981).

20. D. Fried, R. E. Glena, J. D. B. Featherstone, and W. Seka, "Nature of light scattering in dental enamel and dentin at visible and near-infrared wavelengths," Appl. Opt. 34, 1278-1285 (1995).

21. D. Fried, J. D. B. Featherstone, R. E. Glena, B. Bordyn, and W. D. Seka, "Light-scattering properties of dentin and enamel at 543, 632, and 1053 nm," Proc. Soc. Photo-Opt. Instrum. Eng. 1880, 240-245 (1993). 